\title{
Semantic gradients and interference with sorting according to color, spatial position, and numerosity
}

\author{
LESLIE A. FOX \\ Instructional Systems Design, Issaquah, Washington 98027 \\ and \\ RONALD E. SHOR \\ University of New Hampshire, Durham, New Hampshire 09824
}

\begin{abstract}
Stimulus cards in three sets of decks were sorted according to color, spatial position, and numerosity, respectively. Within each set of decks, decks differed as to the semantic relatedness of irrelevant words or symbols to the criterion properties for sorting. Mean sorting times for the 112 subjects were inversely related to the semantic distances between the irrelevant information and the sorting criteria. These results for nonverbal classification responses are compared with the results of an earlier study in which comparable stimuli were classified verbally.
\end{abstract}

It is almost universally found that, when asked to name colors, a person performs the task less well when the colors are those of inks used to print incongruous color names than when the colors appear in nonmeaningful contexts, such as plain patches. The two stimulus conditions of this reliable phenomenon are frequently called the color-word and color tests, respectively.

In elaborating on the color-word test, Klein (1964) found that the interference with color naming caused by irrelevant stimulus attributes follows a gradient that is a function of the semantic relationship of the irrelevant attributes to the color names. For example, Klein's subjects took more time to name the ink colors of color-associated nouns printed in incongruous colors (e.g., grass printed in red ink) than to name the ink colors of noncolor-associated common words. Fox, Shor, and Stcinman (1971) showed that analogous semantic gradients of interference occur when numerosities and spatial positions are the stimulus attributes to be named and that the color semantic gradient of interference found by Klein is a remarkably reliable phenomenon.

Morton (1969) obtained evidence indicating that sorting according to numerosity and sorting according to lateral position are subject to semantic gradients of interference. However, Morton's numerosity sorting evidence is inconclusive in two important respects. First, Morton did not compare the effects of number names and Arabic numerals under equivalent conditions and, second, in labeling the sorting bins with Arabic numerals, Morton may have biased cognitive processing toward an uncustomary form of mediation. Morton's evidence on position sorting cannot necessarily be generalized to the vertical axis, for the gravity sense provides a basis for the up-down distinction, whereas the right-left distinction has no analogous basis.
In the experiment reported here, subjects sorted stimulus cards according to color, spatial position, and numerosity. Correspondence between criterion attributes and sorting locations was specified without the use of symbols. The experiment was conducted exactly like that of Fox et al. (1971) except for the crucial difference in the nature of the classification response and concomitant minor differences in procedure. In particular, individual stimuli used in the present experiment were identical to stimuli used in the experiment of Fox et al.

\section{METHOD}

\section{Subjects}

The subjects were 112 students enrolled in introductory psychology classes at the University of New Hampshire who received course credit for their participation.

\section{Apparatus}

Individual stimuli were constructed on cards of standard poker playing card size, appearing upright when the cards were oriented horizontally. For each condition within each criterion-attribute set, there was a deck of 24 stimulus cards. In each deck all attributes appeared on equal numbers of cards, and each irrelevant attribute was distributed as evenly as possible over the criterion attributes except where the incongruity condition applied.

In the color portion of the experiment, stimuli consisted of words or groups of letters printed in red, green, blue, or black ink. The words or letter groups appearing in the seven decks were: (a) same color names; (b) different color names (GRAY, WHITE, BROWN, YELLOW); (c) colorassociated nouns (BLOOD, GRASS, SKY, COAL); (d) noncolorassociated common words (HAT, CHAIR, IOOOT, HOUSE); (e) uncommon words (CUD, FLUME, NEWT, MIDGE); (f) consonant groups (HVL, JRKBM, TKKT, TLRLK); and (g) groups of four Os.

Stimuli used in the position portion of the experiment were $5.08 \times 5.08 \mathrm{~cm}$ squares, each containing a word or group of letters printed near the top, bottom, right, or left of the square. The words or letter groups appearing in the 
Table 1

Mean Sorting Times

\begin{tabular}{lccccccccc}
\hline & \multicolumn{10}{c}{ Deck } \\
\cline { 2 - 11 } \multicolumn{1}{c}{ Set } & A & B & C & D & E & F & G & H \\
\hline Color & 19.322 & 18.252 & 17.313 & 17.040 & 17.185 & 16.932 & 16.420 \\
Position & 18.883 & 18.070 & 17.224 & 16.997 & 17.084 & 17.312 & 16.436 & \\
Numerosity & 21.696 & 21.106 & 20.849 & 19.886 & $\underline{19.646}$ & 19.331 & 18.818 & 18.055 \\
& & & & & & & & &
\end{tabular}

Note-All entries are given in seconds. Horizontal lines appearing under entries signify nonsignificant differences $(p>.05)$.

seven decks were: (a) same position names (UP, DOWN, RIGHT, LEFT); (b) different position names (NORTH, SOUTH, EAST, WEST); (c) direction-associated verbs (LIFT, DROP, TURN, FLOW); (d) nondirection-associated common words (IT, GRAY, HEART, BOOK); (e) uncommon words (PI, DIRK, STOAT, TARN); (f) consonant groups (JB, FWXD, TRTDR, RDLN); and $(\mathrm{g})$ groups of four Os.

Stimuli used in the numerosity portion were $5.08 \times 5.08 \mathrm{~cm}$ squares, each containing one to six identical symbols or words arranged in a standard die-face pattern. The symbols or words appearing in the eight decks were: (a) same number names (ONE-SIX); (b) same Arabic numerals (1-6); (c) same Roman numerals (I-VI); (d) different Arabic numerals (7-12); (e) common words (AND, BED, GRASS, TAKE, BLUE, CRY); (f) letters (A-F); (g) abstract symbols; and (h) circles.

For each deck set, sorting locations were indicated by a mat on which appeared white squares on a black ground. For color decks the sorting mat contained four squares arrayed laterally. The position sorting mat contained four squares arrayed like the arms of a cross. The numerosity sorting mat contained six squares arrayed laterally.

\section{Design and Procedure}

Order of presentation of the three sets of decks was completely counterbalanced. Presentation order of the decks in each set was determined by balanced Latin squares.

Throughout all instructions, the experimenter identified sorting locations only by pointing to them. For the color set, the four squares from left to right were specified as the locations for red, green, blue, and black stimuli, respectively. For the position set, specification of sorting locations for the four stimulus positions followed the natural physical correspondence. For the numerosity set, the six squares from left to right were specified as the sorting locations for numerosities of one through six, respectively.

Each subject was instructed to sort each deck as rapidly as possible, trying not to make errors, and not to correct errors. Each subject was allowed to sort with either hand and was required to place cards face down as they were sorted. The datum recorded for each deck sorting was the time elapsing from the placing of the first card to the placing of the last card.

\section{RESULTS}

The results clearly showed the effects on sorting of semantic relations between irrelevant and criterion attributes. Mean sorting times for all decks are shown in Table 1.

All sorting times were subjected to a logarithmic transformation before analysis. In separate analyses of variance, the effect of decks was significant ( $p<$ of variance, the effect of decks was significant ( $p<$ .001 ) in each of the three deck sets. Results of NewmanKeuls analyses are indicated in Table 1; horizontal lines signify nonsignificant differences $(p>.05)$ among mean log times. All significant differences are consistent with semantic gradients of interference.

In comparing these results with the results of Fox et al. (1971), it was found that mean sorting times (untransformed) and mean naming times correlated .974 for color stimuli, .923 for position stimuli, and .988 for numerosity stimuli. For subsequent linear regression analyses, each mean sorting time was multiplied by 3 because each sorting performance was timed over 24 stimuli, whereas each naming performance was timed over 72 stimuli. For color stimuli the slope of the linear regression of mean naming times on tripled mean sorting times was 3.581 , with a $95 \%$ confidence interval from 2.623 to 4.539 . For position stimuli the slope was 1.331 , with a confidence interval from .690 to 1.972 . For numerosity stimuli the slope was 1.525 , with a confidence interval from 1.286 to 1.764. From the confidence intervals, it can be seen that the slope for color is significantly greater than the slope for numerosity, which in turn is significantly greater than onę, while the slope for position is not significantly greater than one. For each stimulus set, the regression slope provides a convenient comparison of the naming and sorting gradients in terms of overall steepness. That is, a slope greater than one indicates that the gradient for naming is steeper than that for sorting.

\section{DISCUSSION}

The results clearly and coherently show that sorting according to color, position, or numerosity is subject to a semantic gradient of interference that depends on the semantic relationship of irrelevant stimulus attributes to criterion attributes.

The use of individual stimuli that were identical to those used by Fox et al. (1971) permits the clearest possible comparison of sorting interference effects with those for naming. The extremely high correlations between mean times for sorting and corresponding mean times for naming provide conclusive evidence that the semantic gradients of interference with sorting are qualitatively and ordinally equivalent to the corresponding gradients of interference with naming. The 
linear regression analyses show that the position naming gradient of interference is not significantly steeper than the position sorting gradient; that the numerosity naming gradient is significantly steeper than the numerosity sorting gradient; and that the color naming gradient is significantly steeper than the color sorting gradient, with the difference in steepness being significantly greater than the difference for the numerosity gradients.

The most straightforward explanation for these findings is based on an hypothesis that the steepness of the sorting gradient for a given concept domain approaches the steepness of the naming gradient to the extent that the concepts in that domain have spatial referents. Position concepts are indisputably spatial in nature and, by the hypothesis, the sorting gradient should closely approach the naming gradient in steepness, as was found. Numerosity concepts have clear but indirect spatial referents, for, although ordinal number concepts correspond to relative spatial locations, numerosities (i.e., cardinal number concepts) are one cognitive step removed. Thus, by the hypothesis, the numerosity sorting gradient should not approach the naming gradient as closely in steepness as in the case of positions, and the fact that the regression slope is significantly greater than one accords with this implication. Color concepts have, at best, only a weak relation to spatial events, and it is likely that the spatial referents of the rainbow sequence of hues play a negligible cognitive role for most people. From the hypothesis, it follows that the color sorting gradient should differ markedly from the naming gradient in steepness, as was found.

\section{REFERENCES}

Fox, L. A., Shor, R. E., \& Steinman, R. J. Semantic gradients and interference in naming color, spatial direction, and numerosity. Journal of Experimental Psychology, $1971,91,59-65$.

KLEIN, G. S. Semantic power measured through the interference of words with color-naming. American Journal of Psychology, 1964, 77, 576-588.

Morton, J. Categories of interference: Verbal mediation and conflict in card sorting. British Journal of Psychology, 1969, 60, 329-346.

(Received for publication October 20, 1975.) 